\title{
Global gene expression profiling of Plasmodium falciparum in response to the anti-malarial drug pyronaridine
}

\author{
Kanyanan Kritsiriwuthinan ${ }^{1,2}$, Sastra Chaotheing ${ }^{1}$, Philip J Shaw ${ }^{1 *}$, Chayaphat Wongsombat ${ }^{1}$, \\ Porntip Chavalitshewinkoon-Petmitr ${ }^{2}$ and Sumalee Kamchonwongpaisan ${ }^{{ }^{*}}$
}

\begin{abstract}
Background: Pyronaridine (PN) and chloroquine (CQ) are structurally related anti-malarial drugs with primarily the same mode of action. However, PN is effective against several multidrug-resistant lines of Plasmodium falciparum, including CQ resistant lines, suggestive of important operational differences between the two drugs.

Methods: Synchronized trophozoite stage cultures of P. falciparum strain K1 (CQ resistant) were exposed to 50\% inhibitory concentrations $\left(\mathrm{IC}_{50}\right)$ of $\mathrm{PN}$ and $\mathrm{CQ}$, and parasites were harvested from culture after 4 and 24 hours exposure. Global transcriptional changes effected by drug treatment were investigated using DNA microarrays.

Results: After a $4 \mathrm{~h}$ drug exposure, PN induced a greater degree of transcriptional perturbation (61 differentially expressed features) than CQ (10 features). More genes were found to respond to $24 \mathrm{~h}$ treatments with both drugs, and 461 features were found to be significantly responsive to one or both drugs across all treatment conditions. Filtering was employed to remove features unrelated to primary drug action, specifically features representing genes developmentally regulated, secondary stress/death related processes and sexual stage development. The only significant gene ontologies represented among the 46 remaining features after filtering relate to host exported proteins from multi-gene families.
\end{abstract}

Conclusions: The malaria parasite's molecular responses to PN and CQ treatment are similar in terms of the genes and pathways affected. However, PN appears to exert a more rapid response than CQ. The faster action of PN may explain why PN is more efficacious than $C Q$, particularly against $C Q$ resistant isolates. In agreement with several other microarray studies of drug action on the parasite, it is not possible, however, to discern mechanism of drug action from the drug-responsive genes.

Keywords: Pyronaridine, Chloroquine, microarray, gene expression, Plasmodium falciparum

\section{Background}

Malaria is a parasitic disease accounting for almost one million deaths per year, mostly among children in SubSaharan Africa, Asia, Central and South America [1]. During the last two decades, the incidence of malaria has remained unacceptably high. A major factor in the continuing burden of malaria is the spread of parasites resistant to front-line anti-malarials such as chloroquine

\footnotetext{
* Correspondence: philip@biotec.or.th; sumaleek@biotec.or.th ${ }^{1}$ National Center for Genetic Engineering and Biotechnology (BIOTEC), National Science and Technology Development Agency (NSTDA), Thailand Science Park, Pathumthani 12120, Thailand

Full list of author information is available at the end of the article
}

and sulphadoxine/pyrimethamine [2], and perhaps in the near future, artemisinin [3]. New anti-malarial drugs with different modes of action are needed to overcome drug-resistant parasites.

Pyronaridine (PN) is a Mannich base 9-anilinoacridine structurally related to chloroquine (CQ) (Figure 1). PN was one of the earliest synthetic anti-malarial drugs first developed in China in the late 1970s [4,5]. It is a highly effective blood schizonticidal agent, even against multidrug-resistant lines of Plasmodium falciparum [6,7]. In addition to its schizonticidal activity, it has been shown to possess an in vitro gametocytocidal effect in $P$. falciparum [8]. It is also effective against Plasmodium vivax 

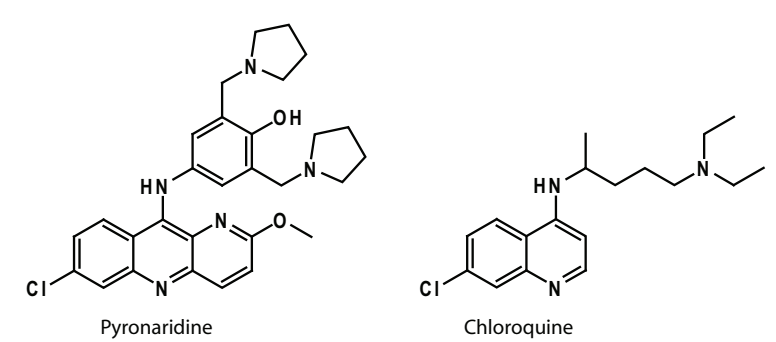

Figure 1 Chemical structures of Pyronaridine (PN) and Chloroquine (CQ).

[9], and Plasmodium ovale and Plasmodium malariae [10]. Furthermore, it has been demonstrated to be effective in Phase III human trials for treating P. falciparum malaria in SE Asia and Africa [11]. Despite extensive reports of its highly potent anti-malarial activity, few studies have been conducted to determine the drug's mechanism of action. Based on the fact that 9-anilinoacridines are potent anti-cancer drugs that target DNA topoisomerase II (TOPO II), it was proposed that PN acted in a similar manner by inhibiting parasite TOPO II [12]. However, it was later shown that parasite TOPO II inhibition by PN is insignificant in situ [13]. Auparakkitanon et al [14] later demonstrated that the primary mode of action of $\mathrm{PN}$ is similar to that of $\mathrm{CQ}$, namely inhibition of $\alpha$-haematin formation, enhanced haematininduced red blood cell lysis and interference with glutathione-dependent heme degradation. The hypothesis of shared mode of action is further supported by the electron microscopic examination of PN-treated P. falciparum from an infected Aotus (owl monkey) [15], which revealed that like $C Q$, the earliest morphological change induced by $\mathrm{PN}$ is the appearance of abnormal vesicles in the food vacuole. Despite the hypothesized similarity of drug action, CQ-resistant field isolates are not crossresistant to $\mathrm{PN}$, implying that there are some important pharmacological differences between the two drugs [7]. From the available information, it is not known whether the differences between CQ and PN efficacy exist because of differences in mode of action, or differences in cellular resistance mechanisms, e.g., to what extent $\mathrm{PN}$ is a substrate for PfCRT-mediated transport compared with CQ.

In this study, a better understanding of the mechanism of action of PN was sought, including the reason why $\mathrm{PN}$ is so effective against CQ-resistant parasites, despite the proposed similarity of drug action for PN and CQ. To help answer these questions, DNA microarrays were employed to measure global gene expression changes of a CQ-resistant strain of P. falciparum in response to individual $50 \%$ inhibitory concentrations of $\mathrm{PN}$ and CQ. There is extensive overlap in the genes affected by both drugs, although PN appears to elicit a more rapid response by the parasite than CQ. The majority of genes changing expression in response to both drugs are likely to be the result of developmental arrest and secondary stress/death related cellular processes; hence, there are very few genes which can be considered as specifically PN and CQ drug responsive.

\section{Methods}

\section{Microarray construction}

DNA microarrays were used containing 8,088 70-mer oligonucleotide probes representing the majority of annotated open reading frames (ORF) as listed in PlasmoDB 4.4 [16]. The oligo probes were printed on polylysine coated glass slides using a new generation ultra fast, linear servo driven DeRisi microarrayer controlled by an ArrayMaker software [17].

\section{Parasite culture and drug treatment}

K1 (chloroquine-resistant) strain of P. falciparum was used for all experiments. This strain was selected since it is representative of the CQ-resistant parasite population where PN is likely to be employed as an anti-malarial in the future. Moreover, PN resistant parasites have not yet been isolated from the field, nor are there any reports of laboratory resistance for $P$. falciparum. Parasites were cultured in vitro as described previously in [18]. Culture synchronization was obtained by lysing mature parasites with $5 \%$ sorbitol for two consecutive cell cycles [19]. Pyronaridine (PN) (a gift from Simon Croft, London School of Hygiene and Tropical Medicine, UK) and chloroquine (CQ) (Sigma-Aldrich) were dissolved in dimethylsulfoxide (DMSO). The 50\% inhibitory concentrations $\left(\mathrm{IC}_{50}\right)$ of each drug against $\mathrm{K} 1$ parasites were determined by the $\left({ }^{3} \mathrm{H}\right)$-hypoxanthine incorporation method [20] with minor modifications. The $\mathrm{IC}_{50}$ values from triplicate biological replicates (with three technical replicates in each) for PN and CQ were $11 \pm 2.0 \mathrm{nM}$ and $276 \pm 38 \mathrm{nM}$ respectively, confirming the PN-sensitive and CQ-resistant phenotypes of the $\mathrm{K} 1$ strain parasites. The $\mathrm{IC}_{50}$ values determined for the K1 strain are very similar to those reported previously [6].

For DNA microarray experiments, a synchronized culture of early trophozoite parasites (22-24 h post-invasion) was treated with the $\mathrm{IC}_{50}$ concentration of each drug (11 nM for PN, and $280 \mathrm{nM}$ for CQ), and the final concentration of DMSO was $0.1 \%(\mathrm{v} / \mathrm{v})$. For each treatment, three culture plates $(90 \times 15 \mathrm{~mm})$ were established, each containing 1.25\% haematocrit and 15-20\% parasitaemia in a total volume of $17 \mathrm{ml}$. The control was a parasite culture (also synchronized and at the same stage as those used for drug treatment) containing $0.1 \%(\mathrm{v} / \mathrm{v})$ DMSO, but lacking drug. Parasites were 
exposed to each drug for 4 and $24 \mathrm{~h}$, and after each time point, parasites were harvested and liberated from erythrocytes by saponin lysis. Drug treatment was performed on 3-6 replicates. For identification of developmentally-regulated transcripts, 3 biological replicates of synchronized cultures of parasites at ring (8-10 h postinvasion), trophozoite (22-24 h post-invasion) and schizont (38-40 h post-invasion) stages were harvested for total RNA extraction.

\section{Total RNA extraction and CDNA labeling}

Total RNA was extracted from parasites using TRIzol reagent (Invitrogen) in accordance with the manufacturer's recommendations. For each cDNA labeling reaction, 10-15 $\mu \mathrm{g}$ of total RNA were annealed with $5 \mu \mathrm{g}$ of oligo $(\mathrm{dT})_{21}$ and reverse transcribed to produce aminoallyl-dUTP(Ambion)-containing cDNA using Stratascript $^{\mathrm{TM}}$ reverse transcriptase (Stratagene). The cDNA from the drug-treated RNA preparations was then labelled with monoreactive Cy5, while the cDNA from control untreated RNA samples was labelled with Cy3. To identify transcripts related to parasite development, cDNA obtained from synchronized parasites at ring, trophozoite and schizont stages were individually labelled with Cy5. A pooled reference RNA was made from the three stage samples mixed in equal proportions. The pooled reference cDNA was labelled with $\mathrm{Cy} 3$.

\section{Microarray hybridization}

Purified Cy3- and Cy5-labelled cDNA were mixed in a Short Oligo Hybridization Solution (Corning Inc.), and hybridized to $P$. falciparum spotted 70-mer oligo microarrays for $16-18 \mathrm{~h}$ at $45^{\circ} \mathrm{C}$. Arrays were washed at room temperature in a solution of $2 \times$ SSC $1 \times$ (Sodium Saline Citrate) containing $0.2 \%$ SDS, and then in $0.1 \times$ SSC. Then arrays were dried by centrifugation and immediately scanned using a ScanArray 4000B microarray scanner (Packard/Perkin Elmer, USA) or a GenePix Pro 4000 scanner according to the manufacturer's instructions. Images were analysed using ScanAlyze software [21] or Genepix Pro to obtain hybridization intensity outputs.

\section{Microarray data analysis}

The raw intensity data from both channels were preprocessed as described previously in [22], and normalized within arrays using the global lowess algorithm and across replicate arrays from the same experiment in the Aroma package [23] run in $\mathrm{R} 2.8 .1$ project environment [24]. All microarray data and details of experimental design were submitted to the NCBI Gene Expression Omnibus (GEO) database and are available under series accession numbers GSE31109, GSE30867 and GSE30869.
After normalization, array features with less than three experimental values were excluded from further analyses. For study of developmentally regulated transcripts, lists were made of the average normalized $\log _{2}$ change in expression of all retained microarray features for the K1 ring, trophozoite and schizont stages $(4625,4651$, and 4640 features, respectively). These lists were then compared with measurements for the corresponding microarray features from 46 time-points across the HB3 strain parasite life cycle as reported in [25] by calculation of global Pearson correlation coefficients for each K1 developmental sample and HB3 time-point.

Differentially expressed genes were identified from each drug treatment time-point using the Significance Analysis of Microarrays (SAM) programme, Excel plugin version 3.05 [26]. Array features showing both a $q$ value of less than $5 \%$ and an expression ratio of $\geq 1.8$ fold in either direction were considered as being differentially expressed (Additional file 1). The SAM algorithm uses nonparametric statistics to calculate $q$-values. The $q$-value for each feature is a measure of the positive false discovery rate (FDR), or how many features showing the same or greater level of differential expression are actually false positives [27]. Heatmap analysis of selected normalized data from array features with a significant change in expression under one or more of the drug treatment conditions was performed using the NeatMap package [28], in which rows (average normalized $\log _{2}$ change in gene expression) were clustered using the nMDS algorithm and columns (drug treatment condition) were clustered by hierarchical average linkage. Gene ontology enrichment analysis of the PN/CQ responsive genes was performed using the GOEAST web tool with default parameters [29]. Data management (filtering of gene lists) and calculation of Pearson correlation coefficients was carried out using Microsoft Excel 2007.

Statistical testing of the overlap of array features common to different drug treatments was performed using a Monte Carlo simulation method [30]. The simulation was performed to determine the null distribution of overlapping features occurring by chance among random selections of features. The simulation generated models of possible results by randomly selecting two different sets of features assumed to be the results of drug treatments. Every instance of feature overlap was tallied yielding distributions of possible outcome values. The model was constructed by randomly selecting features with replacement from the pool of 6203 independent features to reconstruct the subsets of features showing significant responses to PN (297) and CQ (205) drug treatments. The number of intersecting features between the two subsets was observed and tallied. The model is defined as thus: 
Let $S$ be a subset of $A$ where $|A|=N$, the total number of features and $|S|=r$, the number of features in the subset $S$. $S$ corresponds to a set of features chosen randomly (without replacement) from $A$ using uniform distribution. Equivalently, this random process is described using the shorthand $S \leftarrow\left(\begin{array}{l}N \\ r\end{array}\right)$. Let $K$ be the total number of simulations. The Monte Carlo simulation is described as:

$$
\begin{aligned}
& \text { for } i=1 \text { to } K: \\
& S 1 \leftarrow\left(\begin{array}{c}
N \\
r 1
\end{array}\right) \\
& S 2 \leftarrow\left(\begin{array}{c}
N \\
r 2
\end{array}\right) \\
& y(i)=S 1 \cap S 2
\end{aligned}
$$

Note that $y(i)$ is a possible outcome of the experiment. Let $Y$ be a random variable representing the intersection between $S 1$ and $S 2$. Let $K$ be the total number of simulations. The probability of each possible intersection event can be calculated by:

$$
P(\gamma=m)=\sum_{j=1}^{K} j \mid y(j)=m
$$

In the PN/CQ Monte Carlo simulation, $K=1 \times 10^{6}$; $\mathrm{N}=6203 ;|S 1|=297$; and $|S 2|=205$ respectively. The simulation was performed using a custom computer script in the Ruby interpreter programme (Additional file 2).

\section{Results}

\section{Experimental design for comparative transcriptomic profiling}

The goal of this study was to identify global transcriptional changes induced by PN and CQ in a CQ-resistant strain of P. falciparum (K1). Synchronized cultures in early trophozoite stages (22-24 h post invasion) were subjected to $\mathrm{IC}_{50}$ concentrations of $\mathrm{PN}(11 \mathrm{nM})$ and CQ $(280 \mathrm{nM})$ for 4 and $24 \mathrm{~h} . \mathrm{IC}_{50}$ drug treatments were used since previous studies of CQ transcriptional response using lower effective drug concentrations did not reveal any drug-induced transcriptional changes [31]. Conversely, very high drug concentrations can lead to developmental arrest which confounds analysis of the drug response (see below). Preliminary study with $30 \mathrm{~min}$ drug exposures did not reveal any significant changes in expression for either PN or CQ (data not shown). The 4 $\mathrm{h}$ time point was chosen to represent an 'early' response to the drug in which we could be reasonably confident of detecting some significant changes in expression. Importantly, the $4 \mathrm{~h}$ time point is also physiologically relevant with respect to pharmacokinetics, since the time taken for PN and CQ to reach their maximum plasma concentrations in patients administered with the drugs are 2-3 $\mathrm{h}$ for PN [32] and 2-6 h for CQ [33]. The $24 \mathrm{~h}$ time point was regarded as a 'late' drug response after which parasites had passed through the schizont stage, which is known to be susceptible to PN and CQ action.

To gauge the global drug-induced transcriptomic changes, DNA microarray experiments were performed in which cDNA samples from drug-treated and nontreated control cultures from each time point were labelled with different cyanine dyes, mixed and hybridized to the same microarray. In total, the dataset comprised nine microarray hybridizations each for PN and CQ.

\section{Comparison of PN and CQ transcriptional responses}

In total, 297 and 205 microarray features were differentially expressed under PN and CQ, respectively. There is an overlap of 40 features which show significant changes in expression to both drugs (Additional file 3). To test whether this overlap is significant, a Monte Carlo simulation was performed to discover the null distribution of overlap occurring by chance for two feature lists with the corresponding number of features made randomly from the total of 6203 features that give robust signals (three or more experimental values) in all $\mathrm{PN}$ and $\mathrm{CQ}$ microarray experiments. The simulation shows that the PN and CQ overlap is highly significant $\left(p \leq 1 \times 10^{-6}\right)$, indicating a significant similarity between the two responses. To determine the biological significance of the overlap between PN and CQ responsive features, the 254 significant features responsive to $24 \mathrm{~h}$ exposure of Pyrimethamine, an antifolate drug with a different mode of action [34] in the same K1 parasite strain were compared with the $P N$ and $C Q$ responsive features. The number of overlaps in each case was not significant $(8$ overlapping features for Pyrimethamine/CQ and 9 for Pyrimethamine/PN; Additional file 3).

To compare the global transcriptional responses of parasites to $\mathrm{PN}$ and $\mathrm{CQ}$ after different periods of exposure, clustering and heatmap analyses were performed on the 461 features showing significant differential expression under at least one drug treatment (Figure 2). The overall transcriptional response patterns of the PN $4 \mathrm{~h}, \mathrm{PN} 24 \mathrm{~h}$ and CQ $24 \mathrm{~h}$ treatments are similar to one another, which are confirmed by the global Pearson correlations (Table 1). The overall change in gene expression is more modest after $4 \mathrm{~h} \mathrm{CQ}$ exposure compared with the others. In terms of significant differentially expressed features, far fewer were found after CQ $4 \mathrm{~h}$ exposure (10) than other treatments $(61,239$ and 195 for PN $4 \mathrm{~h}, \mathrm{PN} 24 \mathrm{~h}$ and CQ $24 \mathrm{~h}$, respectively; Additional file 1). 


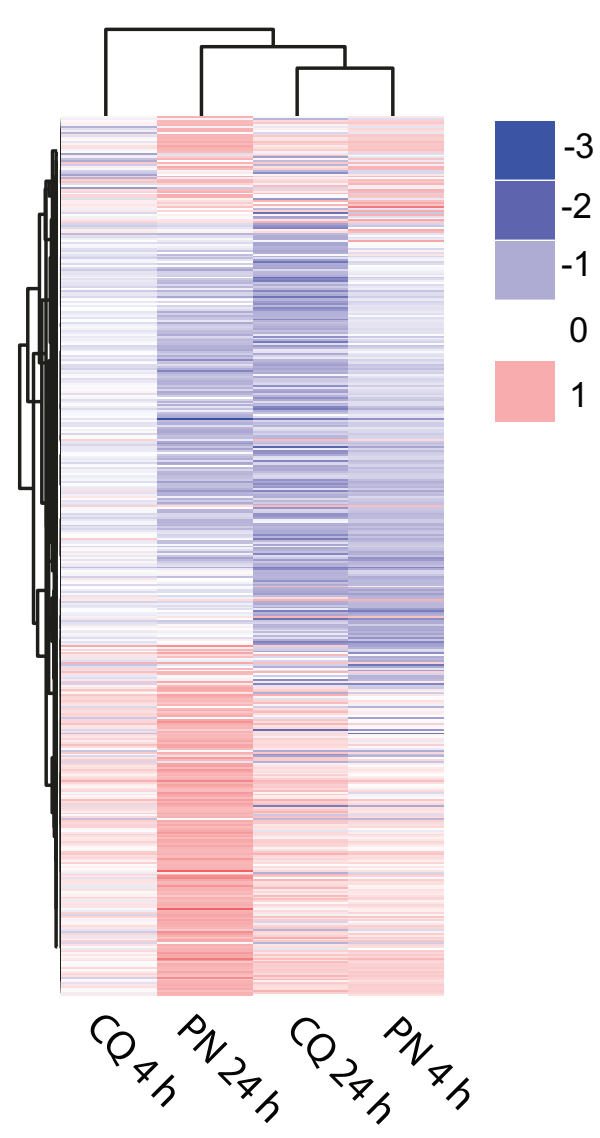

Figure 2 All PN and CQ significant responsive features. The data from 463 features showing significant changes in expression identified from microarray analysis were clustered according to the average gene expression changes as rows and the drug treatments as columns. The colour key indicates the average $\log _{2}$ fold changes in expression.

Although many genes with significant changes in expression after drug treatment were identified, it should be appreciated that drugs typically induce cell cycle arrest, or delay before cell killing, leading to differences in cell stages between drug-treated and control cultures. During normal growth, parasite stages show markedly different gene expression profiles from one another [25,35], and so the gene expression profile of

Table 1 Global Pearson correlations for microarray data

\begin{tabular}{ccccc}
\hline & CQ 4 h & CQ 24 h & PN 4 h & PN 24 h \\
\hline CQ 4 h & 1 & & & \\
\hline CQ 24 h & 0.62 & 1 & & \\
\hline PN 4 h & 0.56 & 0.77 & 1 & \\
\hline PN 24 h & 0.62 & 0.73 & 0.65 & 1 \\
\hline
\end{tabular}

Global Pearson correlation coefficients for each pairing of drug treatment conditions were calculated from the 463 features significantly responsive to $\mathrm{PN}$ and/or $\mathrm{CQ}$ drug-treated parasites will likely differ from control untreated parasites in part because of drug-induced developmental delay. Different anti-malarial drugs induce cell cycle arrest to different extents; for example, lethal concentrations of antifolates do not induce morphological arrest [36]. On the other hand, the developmental arrest induced by cytostatic drugs such as DL- $\alpha$ difluoromethylornithine necessitate complex experimental designs employing multiple time-points of drug-treated and control cultures in order to correct for transcriptional changes relating only to differences in cell stage [37].

To identify the developmentally regulated genes, which could confound identification of PN and CQresponsive genes, RNA samples from drug-free synchronized cultures from ring, trophozoite, and schizont stages were individually labelled and hybridized with a pooled sample from the three stages. The data from this experiment were used to compare the developmental profile of the K1 strain with the high-resolution data for the HB3 strain (Additional file 4). The peak of Pearson correlation between the $\mathrm{K} 1$ trophozoite culture sample (estimated age 22-24 h post infection) and HB3 is maximal at HB3 time-points 20-24 h (Figure 3), indicating a high concordance between the two strains in terms of developmentally regulated transcripts.

After $4 \mathrm{~h}$ drug treatment, $>90 \%$ of parasites were at the trophozoite stage, while after $24 \mathrm{~h}$ drug treatment parasites were at schizont and early ring stages. Therefore, microarray features showing developmental change in the HB3 dataset (1.8 fold or greater change in expression) were filtered out from the lists of features differentially expressed under drug treatment. For the $4 \mathrm{~h}$ drug treatments, features which were found to be HB3 developmentally regulated at any of the time-points 22-28 $\mathrm{h}$ post infection were removed. For the $24 \mathrm{~h}$ drug

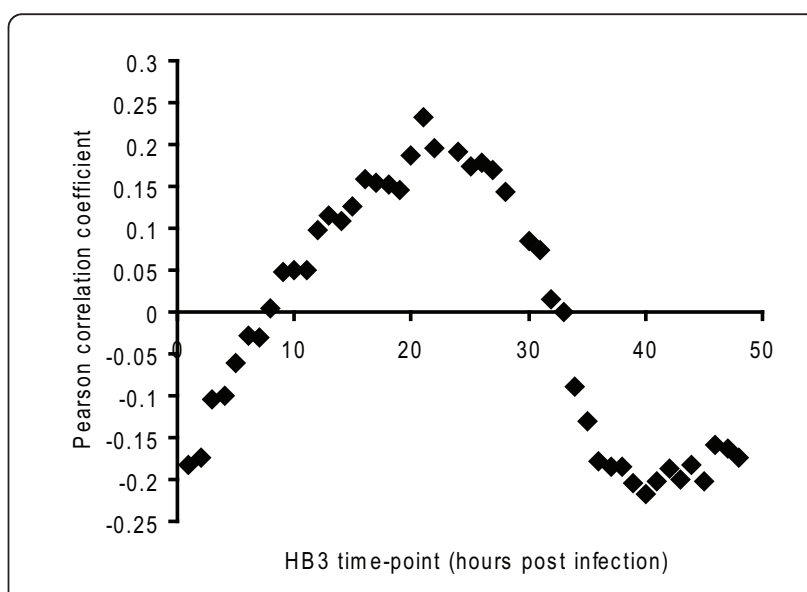

Figure 3 Correlation between K1 Trophozoite (22-24 h) and intraerythrocytic developmental cycle data for strain HB3. 
treatments, features developmentally regulated at any of the HB3 time-points $22-48 \mathrm{~h}$ and $1-4 \mathrm{~h}$ post infection were removed. This filtering, therefore, establishes a subset of differentially expressed features in which features showing expression changes solely due to differences in staging during the course of drug exposure have been removed. In total, there were 145 features after developmental filtering which showed significantly different expression under at least one of the drug exposure conditions tested (Additional file 5).

Developmental arrest induced by drug action is but one confounding factor in the analysis of drug-induced transcriptional profiles. In order to gain insight into drug mechanism of action from microarray data, the primary or direct effects of the drug need to separated from indirect (e.g. general stress response), secondary and bystander effects (reviewed in [38]). A recent P. falciparum microarray study identified a set of 3705 "perturbation" responsive genes which change expression in response to widely different drugs and other stresses [39]. The perturbation gene set was then used to filter out features likely responding to drug in an indirect, or secondary fashion. $72 \mathrm{PN} / \mathrm{CQ}$ responsive features with gene annotations matching the perturbation gene list were removed by this filtering step. Another indirect genetic response to drug action is activation of gametocyte stage genes [40]. $26 \mathrm{PN} / \mathrm{CQ}$ features with gene annotations matching the list of genes up-regulated (pAnova $<0.05$ ) in gametocyte stages [41] were removed by this final filtering step, leaving 46 primary PN/CQ responsive features. The heatmap analysis of these features in Figure 4 shows overall similarity of PN and CQ responses. Inspection of the annotated genes for these features reveals a striking abundance of genes encoding host-exported proteins of different families, i.e. var, stevor, surfin, rifin and phist, which is supported by the significant gene ontology terms (Table 2).

\section{Discussion}

Although PN is well-recognized as a highly potent antimalarial drug against both sensitive and multi-drug resistant lines of malaria parasites, its molecular mechanism of action is not fully understood. Previous studies have demonstrated that its primary mode of action is the same as CQ, namely interaction with haem and subsequent inhibition of $\beta$-haematin assembly. Given that both drugs are structurally related and are proposed to act on the same target(s), it was hypothesized that they may induce similar transcriptomic changes at $\mathrm{IC}_{50}$ concentrations.

The transcriptome of chloroquine-resistant P. falciparum $\mathrm{K} 1$ strain in response to $\mathrm{PN}$ and $\mathrm{CQ}$ exposure was investigated. Similar to previous microarray studies of $P$. falciparum under anti-malarial drug treatment
$[36,42]$, the magnitude of change in expression for most genes in response to CQ and PN was low. Nonetheless, 297 features showed significant change to PN and 205 to CQ. The overlap of 40 features among the PN and CQ features was highly significant, suggestive of a similar genetic response. In contrast, the PN and CQ features overlapping with those responsive to an unrelated drug, Pyrimethamine in the same $\mathrm{K} 1$ parasite strain exposed for 24 hours were not significant. In total, 461 features showed significant changes in response to either or both PN and CQ drugs. A major difficulty in interpreting these data with respect to primary mechanism of drug action is confounding responses not directly related to the mechanism of drug action. Although no delay of parasite development was observed in CQ and PN drug-treated cultures by comparison of morphology, it is known that transcriptional arrest precedes morphological arrest [37]. It should be noted that the term transcriptional arrest relates to a delay in the stage-specific pattern of gene expression within a short time-frame, i. e. the same $48 \mathrm{~h}$ developmental cycle. This phenomenon is probably quite distinct from the changes occurring in recrudescing parasites in the longer time-scale of a clinical treatment period (3-4 days). Such a delay in the developmental programme of gene expression may, therefore, have a confounding effect on identification of drug-specific responders. A conservative approach was taken to address this confounding effect, in which all stage-specific transcripts from the corresponding developmental stages during the different drug treatment periods were removed. This approach, however, could lead to removal of genes which are primary drug responsive, yet developmentally regulated.

Besides the primary effects of PN and CQ treatment, i. e. inhibition of $\alpha$-haematin formation and glutathionedependent haem degradation, secondary/downstream effects of these drugs on the parasite have been described. At very high concentrations, CQ and other quinoline drugs disrupt vesicular transport $[43,44]$. Moreover, CQ inhibits translation [45], disrupts mitochondrial membrane potential and induces DNA fragmentation [46]. The PN and CQ responsive microarray features with gene annotations matching the perturbation dataset [39] could be considered secondary responses. The gene annotations encompass a variety of cellular functions, although none are significantly associated with the aforementioned CQ secondary effects. Microarray features corresponding to the Pfcrt gene, which mediates resistance to $C Q$, showed significant changes in expression in response to PN and CQ. However, the Pfcrt gene is present in the perturbation dataset and cannot be considered as a primary PN or CQ responsive gene. Moreover, no significant changes in expression were found for features corresponding to 


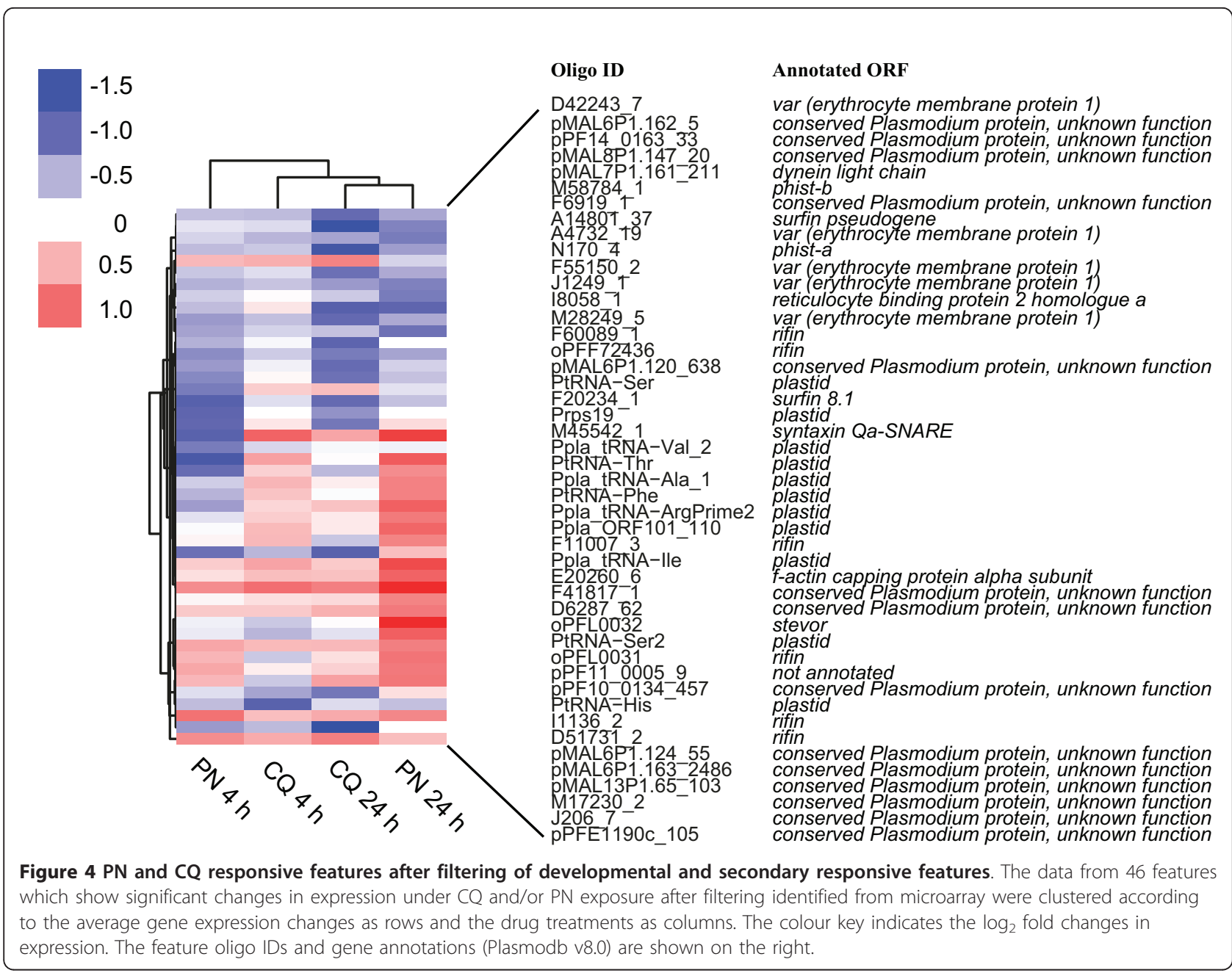

other CQ and quinoline drug transporter genes reported in the literature (Pfmdr1, Pfmdr 2, Pfmrp1, Pfmrp2 and Pfnhe1).

After filtering by comparison with the perturbation dataset and removal of genes known to be up-regulated in gametogenesis, the remaining $46 \mathrm{PN}$ and $\mathrm{CQ}$ responsive features showed a significant over-representation of genes encoding host-exported proteins. These genes belong to large gene families, including var, stevor, surfin, rifin and phist. A previous study of the transcriptional response of $P$. falciparum under $\mathrm{IC}_{50} \mathrm{CQ}$ treatment showed that 36 genes could be considered as

Table 2 Enriched Gene ontology terms represented by the 46 PN/CQ features after filtering

\begin{tabular}{|c|c|c|c|c|c|c|c|c|c|}
\hline GO ID & Ontology & Term & $q$ & $\mathrm{~m}$ & $\mathrm{~T}$ & $\mathrm{~K}$ & genes & log_odds_ratio & $p$ \\
\hline GO:0005576 & cellular_component & $\begin{array}{l}\text { extracellular } \\
\text { region }\end{array}$ & 7 & 221 & 2198 & 10 & $\begin{array}{l}\text { PFA0765c//MAL7P1.57//PFE0065w//PFI0005w// } \\
\text { PFF0855c//PFL2640c//PFL2620w }\end{array}$ & 2.7995 & 0.000272 \\
\hline GO:0018995 & cellular_component & host & 7 & 220 & 2198 & 10 & $\begin{array}{l}\text { PFA0765c//MAL7P1.57//PFE0065w//PFI0005w// } \\
\text { PFF0855c//PFL2640c//PFL2620w }\end{array}$ & 2.806043 & 0.000272 \\
\hline GO:0020002 & cellular_component & $\begin{array}{l}\text { host cell plasma } \\
\text { membrane }\end{array}$ & 5 & 190 & 2198 & 10 & $\begin{array}{l}\text { PFA0765c//MAL7P1.57//PFI0005w//PFF0855c// } \\
\text { PFL2640c }\end{array}$ & 2.53212 & 0.01505 \\
\hline GO:0033643 & cellular_component & host cell part & 7 & 220 & 2198 & 10 & $\begin{array}{l}\text { PFA0765c//MAL7P1.57//PFE0065w//PFI0005w// } \\
\text { PFF0855c//PFL2640c//PFL2620w }\end{array}$ & 2.806043 & 0.000272 \\
\hline
\end{tabular}

Significantly enriched gene ontology terms were identified using the GOEAST tool. The top four significant terms are shown. GO ID is the identifier for the GO term in the Gene Ontology Project, Ontology represents the ontology category the GO term belongs to, term is the GO definition, $\mathrm{q}$ is the number of genes associated with the same GO ID in the data analyzed, $m$ is the total number of genes associated with the listed GO ID in the genome, $t$ is the total number of annotated genes for Plasmodium falciparum, $\mathrm{k}$ is the total number of features with gene annotations considered for analysis, genes are the Gene IDs sharing the same significant GO ID, $\log _{-}$odds_ratio is the $\log _{2}$ odds ratio of the GO ID enrichment and $\mathrm{p}$ is the FDR-adjusted $p$-value for significance. 
primary CQ responsive, with many more genes showing changes in expression because of differences in staging and secondary effects [47]. Of the 36 primary CQ genes reported in [47], two rifin genes (PF10_0006 and PF10_0404) are also present among the annotated genes represented by the $46 \mathrm{PN} / \mathrm{CQ}$ responsive features after filtering. Although the data are suggestive of primary $\mathrm{PN}$ and CQ responsive genes, other perturbations such as antifolates [36], and the bisthiazolium compound T4 [40] also induce changes in expression of host-exported proteins of multi-gene families, albeit for separate genes. Despite some common features of transcriptional regulation, including epigenetic switching mechanisms and sharing of transcriptional activators [48], very little is known of how the expressions of these gene families are modulated by environmental perturbation. Given that different drugs exert responses in these gene families, their roles as potential mediators of drug resistance should be investigated further.

Comparison of the drug-responsive genes shows that PN appears to exert a faster-acting transcriptional perturbation than CQ. From the four drug treatment conditions tested in this study, the PN $4 \mathrm{~h}$ treatment was globally more similar to the CQ 24 h treatment than CQ $4 \mathrm{~h}$. Similarities between the PN and CQ transcriptional responses were also noted in overlapping genes and functional categories, which support the hypothesis that the genes responsive to the two drugs are similar. It should be noted that because $\mathrm{PN}$ is far more potent than $\mathrm{CQ}$ against the $\mathrm{K} 1$ strain, much higher concentrations of the latter drug were needed to produce similar biological effects. Therefore, the differences in cytosolic concentrations between PN and CQ may contribute to some of the minor observed differences between the $\mathrm{PN}$ and CQ transcriptional responses. A simple explanation for why PN is faster acting and thus more effective than $C Q$, especially against $C Q$-resistant parasites, can be made based on the known chemical properties. $\mathrm{PN}$ is more hydrophobic than $\mathrm{CQ}$, with a $\log \mathrm{P}$ value comparable to amodiaquine $[49,50]$ and thus the uptake into the food vacuole, the site of action, will be faster for PN. Furthermore, PN has an extra highly basic group, which means it may be protonated more readily and thus accumulate more rapidly in the food vacuole than $C Q$.

\section{Conclusions}

This study is the first to report global perturbations on $P$. falciparum gene expression in response to $\mathrm{IC}_{50}$ concentration of PN. Since the primary modes of action for $\mathrm{PN}$ and CQ are thought to be the same, expression changes induced by CQ were studied for comparison. The genetic response to $\mathrm{PN}$ and $\mathrm{CQ}$ are similar, although PN appears to exert a faster and more pronounced effect than CQ since the transcriptomic changes are discernable after a $4 \mathrm{~h}$ exposure of $\mathrm{PN}$, whereas a longer exposure is needed for a comparable effect with CQ. It is not possible, however, to relate the drug-responsive genes with the mode of action of these drugs, since the majority of genes changing expression can be related with drug-induced developmental arrest and general response to perturbation.

\section{Additional material}

\section{Additional file 1: PN and CQ significant responsive features.}

Microarray features showing significant changes in expression called by the Statistical Analysis of Microarrays algorithm under each PN and CQ treatment condition.

Additional file 2: Null distribution of overlapping microarray features from PN and CQ drug treatments determined by Monte Carlo modelling.

Additional file 3: Comparison of features showing significant changes in expression under different drug treatments.

Additional file 4: Comparison of transcriptional changes between K1 and HB3 intraerythrocytic stages.

Additional file 5: Filtering of PN and CQ features (i) developmentally regulated across treatment periods identified by matching to HB3 dataset (ii) perturbation responsive identified from Hu et al. dataset (iii) upregulated in gametocyte stages, identified from Young et al. dataset. See Materials and methods for details.

\section{Acknowledgements}

We thank Drs. Joseph Derisi and Jennifer Shock at University of San Francisco for providing the oligo-set, training and facilities for the production of DNA microarray slides through generous support from the Howard Hughes Medical Institute (HHMI, USA). We would also like to thank Dr. Simon Croft at the London School of Hygiene and Tropical Medicine, UK for generously providing pyronaridine, Dr. Sissades Tongsima and Supasak Kulawonganunchai (Bioinformatics and Statistics laboratory, BIOTEC, Thailand) for their assistance in Monte Carlo statistical analysis and Ms. Mayurachat Poopha and Ms. Roonglawan Rattanajak for technical assistance in malaria culture. We are indebted to Professors Yongyuth Yuthavong and Prapon Wilairat for their invaluable advice and for critical review of the manuscript. This work is supported by Thailand Graduate Institute of Science and Technology (TGIST) under NSTDA to KK, UNICEF/UNDP/World Bank/WHO special Programme for Research and Training in Tropical Diseases (TDR) and Thailand-TDR Programme to SK. SK is also an international research scholar of HHMI. PJS is funded by a grant from the Bill \& Melinda Gates Foundation through the Grand Challenges Explorations initiative.

\section{Author details}

${ }^{1}$ National Center for Genetic Engineering and Biotechnology (BIOTEC), National Science and Technology Development Agency (NSTDA), Thailand Science Park, Pathumthani 12120, Thailand. 'Department of Protozoology, Faculty of Tropical Medicine, Mahidol University, Bangkok 10400, Thailand.

\section{Authors' contributions}

KK performed drug-response microarray experiments, data processing and analysis. CW performed cell-cycle microarray experiments and processed data. SC and PJS analysed data, prepared figures and wrote the manuscript. PCP and SK analysed data and helped drafting and editing of the manuscript. All authors read and approved the manuscript.

\section{Competing interests}

The authors declare that they have no competing interests. 
Received: 10 May 2011 Accepted: 18 August 2011

Published: 18 August 2011

\section{References}

1. World Malaria Report 2010. [http://www.who.int/malaria/ world_malaria_report_2010/en/index.html].

2. Muller IB, Hyde JE: Antimalarial drugs: modes of action and mechanisms of parasite resistance. Future Microbiol 2010, 5:1857-1873.

3. Dondorp AM, Nosten F, Yi P, Das D, Phyo AP, Tarning J, Lwin KM, Ariey F, Hanpithakpong W, Lee SJ, Ringwald P, Silamut K, Imwong M, Chotivanich K, Lim P, Herdman T, An SS, Yeung S, Singhasivanon P, Day NP, Lindegardh N, Socheat D, White NJ: Artemisinin resistance in Plasmodium falciparum malaria. N Engl J Med 2009, 361:455-467.

4. Zheng XY, Chen C, Gao FH, Zhu PE, Guo HZ: Synthesis of new antimalarial drug pyronaridine and its analogues (author's transl). Yao Xue Xue Bao 1982, 17:118-125.

5. Zheng XY, Xia Y, Gao FH, Chen C: [Synthesis of 7351, a new antimalarial drug (author's transl)]. Yao Xue Xue Bao 1979, 14:736-737.

6. Elueze El, Croft SL, Warhurst DC: Activity of pyronaridine and mepacrine against twelve strains of Plasmodium falciparum in vitro. J Antimicrob Chemother 1996, 37:511-518.

7. Kurth F, Pongratz $P$, Belard S, Mordmuller B, Kremsner PG, Ramharter M: In vitro activity of pyronaridine against Plasmodium falciparum and comparative evaluation of anti-malarial drug susceptibility assays. Malar J 2009, 8:79.

8. Chavalitshewinkoon-Petmitr $P$, Pongvilairat $G$, Auparakkitanon $S$, Wilairat $P$ : Gametocytocidal activity of pyronaridine and DNA topoisomerase II inhibitors against multidrug-resistant Plasmodium falciparum in vitro. Parasitol Int 2000, 48:275-280.

9. Druilhe P, Brasseur P, Blanc C, Makler M: Improved assessment of Plasmodium vivax response to antimalarial drugs by a colorimetric double-site plasmodium lactate dehydrogenase antigen capture enzyme-linked immunosorbent assay. Antimicrob Agents Chemother 2007, 51:2112-2116.

10. Ringwald P, Bickii J, Same-Ekobo A, Basco LK: Pyronaridine for treatment of Plasmodium ovale and Plasmodium malariae infections. Antimicrob Agents Chemother 1997, 41:2317-2319.

11. Tshefu AK, Gaye O, Kayentao K, Thompson R, Bhatt KM, Sesay SS, Bustos DG, Tjitra E, Bedu-Addo G, Borghini-Fuhrer I, Duparc S, Shin CS, Fleckenstein L: Efficacy and safety of a fixed-dose oral combination of pyronaridine-artesunate compared with artemether-lumefantrine in children and adults with uncomplicated Plasmodium falciparum malaria: a randomised non-inferiority trial. Lancet 2010, 375:1457-1467.

12. Chavalitshewinkoon P, Wilairat P, Gamage S, Denny W, Figgitt D, Ralph R: Structure-activity relationships and modes of action of 9-anilinoacridines against chloroquine-resistant Plasmodium falciparum in vitro. Antimicrob Agents Chemother 1993, 37:403-406.

13. Auparakkitanon S, Wilairat P: Cleavage of DNA induced by 9anilinoacridine inhibitors of topoisomerase II in the malaria parasite Plasmodium falciparum. Biochem Biophys Res Commun 2000, 269:406-409.

14. Auparakkitanon S, Chapoomram S, Kuaha K, Chirachariyavej T, Wilairat P: Targeting of hematin by the antimalarial pyronaridine. Antimicrob Agents Chemother 2006, 50:2197-2200.

15. Kawai S, Kano S, Chang C, Suzuki M: The effects of pyronaridine on the morphology of Plasmodium falciparum in Aotus trivirgatus. Am J Trop Med Hyg 1996, 55:223-229.

16. Aurrecoechea C, Brestelli J, Brunk BP, Dommer J, Fischer S, Gajria B, Gao X Gingle A, Grant G, Harb OS, Heiges M, Innamorato F, lodice J, Kissinger JC, Kraemer E, Li W, Miller JA, Nayak V, Pennington C, Pinney DF, Roos DS, Ross C, Stoeckert CJ Jr, Treatman C, Wang H: PlasmoDB: a functional genomic database for malaria parasites. Nucleic Acids Res 2009, 37 D539-543.

17. Making Arrays. [http://derisilab.ucsf.edu/data/microarray/software.html].

18. Trager W, Jensen JB: Human malaria parasites in continuous culture. Science 1976, 193:673-675.

19. Lambros C, Vanderberg JP: Synchronization of Plasmodium falciparum erythrocytic stages in culture. J Parasitol 1979, 65:418-420.

20. Desjardins RE, Canfield CJ, Haynes JD, Chulay JD: Quantitative assessment of antimalarial activity in vitro by a semiautomated microdilution technique. Antimicrob Agents Chemother 1979, 16:710-718.
21. Microarray Image Analysis ScanAlyze Download. [http://rana.lbl.gov/ EisenSoftware.htm].

22. Shaw PJ, Ponmee N, Karoonuthaisiri N, Kamchonwongpaisan S, Yuthavong Y: Characterization of human malaria parasite Plasmodium falciparum elF4E homologue and mRNA 5' cap status. Mol Biochem Parasitol 2007, 155:146-155.

23. aroma - An R Object-oriented Microarray Analysis Environment. [http:// www.maths.lth.se/publications/].

24. The R Project for Statistical Computing. [http://www.r-project.org/].

25. Bozdech Z, Llinas M, Pulliam BL, Wong ED, Zhu J, DeRisi JL: The transcriptome of the intraerythrocytic developmental cycle of Plasmodium falciparum. PLoS Biol 2003, 1:E5.

26. Tusher VG, Tibshirani R, Chu G: Significance analysis of microarrays applied to the ionizing radiation response. Proc Natl Acad Sci USA 2001, 98:5116-5121.

27. Storey JD, Tibshirani R: Statistical significance for genomewide studies. Proc Natl Acad Sci USA 2003, 100:9440-9445.

28. Rajaram $\mathrm{S}$, Oono Y: NeatMap-non-clustering heat map alternatives in $\mathrm{R}$. BMC Bioinformatics 2010, 11:45.

29. Zheng Q, Wang XJ: GOEAST: a web-based software toolkit for Gene Ontology enrichment analysis. Nucleic Acids Res 2008, 36:W358-363.

30. Fishman GS: Monte Carlo: concepts, algorithms, and applications, Corr. New York: Springer; 21997.

31. Jiang H, Patel JJ, Yi M, Mu J, Ding J, Stephens R, Cooper RA, Ferdig MT, Su XZ: Genome-wide compensatory changes accompany drug- selected mutations in the Plasmodium falciparum crt gene. PLoS One 2008, 3 : e2484.

32. Ramharter M, Kurth F, Schreier AC, Nemeth J, Glasenapp I, Belard S, Schlie M, Kammer J, Koumba PK, Cisse B, Mordmuller B, Lell B, Issifou S, Oeuvray C, Fleckenstein L, Kremsner PG: Fixed-dose pyronaridineartesunate combination for treatment of uncomplicated falciparum malaria in pediatric patients in Gabon. J Infect Dis 2008, 198:911-919.

33. Lee SJ, McGready R, Fernandez C, Stepniewska K, Paw MK, Viladpainguen SJ, Thwai KL, Villegas L, Singhasivanon P, Greenwood BM, White NJ, Nosten F: Chloroquine pharmacokinetics in pregnant and nonpregnant women with vivax malaria. Eur J Clin Pharmacol 2008, 64:987-992.

34. Ponmee N: Study on mechanisms of Pyrimethamine resistance in Plasmodium falciparum by microarray analysis. PhD Thesis Mahidol University, Biochemistry; 2006.

35. Le Roch KG, Zhou Y, Blair PL, Grainger M, Moch JK, Haynes JD, De La Vega P, Holder AA, Batalov S, Carucci DJ, Winzeler EA: Discovery of gene function by expression profiling of the malaria parasite life cycle. Science 2003, 301:1503-1508

36. Ganesan K, Ponmee N, Jiang L, Fowble JW, White J, Kamchonwongpaisan S, Yuthavong Y, Wilairat P, Rathod PK: A genetically hard-wired metabolic transcriptome in Plasmodium falciparum fails to mount protective responses to lethal antifolates. PLOS Pathog 2008, 4:e1000214.

37. van Brummelen AC, Olszewski KL, Wilinski D, Llinas M, Louw Al, Birkholtz LM: Co-inhibition of Plasmodium falciparum Sadenosylmethionine decarboxylase/ornithine decarboxylase reveals perturbation-specific compensatory mechanisms by transcriptome, proteome, and metabolome analyses. J Biol Chem 2009, 284:4635-4646.

38. Brazas MD, Hancock RE: Using microarray gene signatures to elucidate mechanisms of antibiotic action and resistance. Drug Discov Today 2005, 10:1245-1252.

39. Hu G, Cabrera A, Kono M, Mok S, Chaal BK, Haase S, Engelberg K, Cheemadan S, Spielmann T, Preiser PR, Gilberger TW, Bozdech Z: Transcriptional profiling of growth perturbations of the human malaria parasite Plasmodium falciparum. Nat Biotechnol 2010, 28:91-98.

40. Le Roch KG, Johnson JR, Ahiboh H, Chung DW, Prudhomme J, Plouffe D, Henson K, Zhou Y, Witola W, Yates JR, Mamoun CB, Winzeler EA, Vial H: A systematic approach to understand the mechanism of action of the bisthiazolium compound T4 on the human malaria parasite, Plasmodium falciparum. BMC Genomics 2008, 9:513.

41. Young JA, Fivelman QL, Blair PL, de la Vega P, Le Roch KG, Zhou Y, Carucci DJ, Baker DA, Winzeler EA: The Plasmodium falciparum sexual development transcriptome: a microarray analysis using ontology-based pattern identification. Mol Biochem Parasitol 2005, 143:67-79.

42. Natalang O, Bischoff E, Deplaine G, Proux C, Dillies MA, Sismeiro O, Guigon G, Bonnefoy S, Patarapotikul J, Mercereau-Puijalon O, Coppee JY, David PH: Dynamic RNA profiling in Plasmodium falciparum synchronized 
blood stages exposed to lethal doses of artesunate. BMC Genomics 2008, 9:388.

43. Hoppe HC, van Schalkwyk DA, Wiehart UI, Meredith SA, Egan J, Weber BW: Antimalarial quinolines and artemisinin inhibit endocytosis in

Plasmodium falciparum. Antimicrob Agents Chemother 2004, 48:2370-2378.

44. Roberts L, Egan TJ, Joiner KA, Hoppe HC: Differential effects of quinoline antimalarials on endocytosis in Plasmodium falciparum. Antimicrob Agents Chemother 2008, 52:1840-1842.

45. Surolia N, Padmanaban G: Chloroquine inhibits heme-dependent protein synthesis in Plasmodium falciparum. Proc Natl Acad Sci USA 1991, 88:4786-4790.

46. Ch'ng JH, Kotturi SR, Chong AG, Lear MJ, Tan KS: A programmed cell death pathway in the malaria parasite Plasmodium falciparum has general features of mammalian apoptosis but is mediated by clan CA cysteine proteases. Cell Death Dis 2010, 1:e26.

47. Gunasekera AM, Myrick A, Le Roch K, Winzeler E, Wirth DF: Plasmodium falciparum: genome wide perturbations in transcript profiles among mixed stage cultures after chloroquine treatment. Exp Parasitol 2007, 117:87-92.

48. Cui L, Miao J: Chromatin-mediated epigenetic regulation in the malaria parasite Plasmodium falciparum. Eukaryot Cell 2010, 9:1138-1149.

49. Qi J, Wang S, Liu G, Peng H, Wang J, Zhu Z, Yang C: Pyronaridine, a novel modulator of P-glycoprotein-mediated multidrug resistance in tumor cells in vitro and in vivo. Biochem Biophys Res Commun 2004, 319:1124-1131.

50. Warhurst DC, Craig JC, Adagu IS, Meyer DJ, Lee SY: The relationship of physico-chemical properties and structure to the differential antiplasmodial activity of the cinchona alkaloids. Malar J 2003, 2:26.

doi:10.1186/1475-2875-10-242

Cite this article as: Kritsiriwuthinan et al: Global gene expression profiling of Plasmodium falciparum in response to the anti-malarial drug pyronaridine. Malaria Journal 2011 10:242.

\section{Submit your next manuscript to BioMed Central and take full advantage of:}

- Convenient online submission

- Thorough peer review

- No space constraints or color figure charges

- Immediate publication on acceptance

- Inclusion in PubMed, CAS, Scopus and Google Scholar

- Research which is freely available for redistribution

Submit your manuscript at www.biomedcentral.com/submit 